\title{
A Three-Dimensional Shape Description Algorithm Based on Polar-Fourier Transform for 3D Model Retrieval
}

\author{
Dariusz Frejlichowski \\ West Pomeranian University of Technology, Szczecin, \\ Faculty of Computer Science and Information Technology, \\ Zolnierska 49, 71-210, Szczecin, Poland \\ dfrejlichowski@wi.zut.edu.pl
}

\begin{abstract}
In the paper a new 3D shape representation algorithm is proposed - the Polar-Fourier 3D Shape Descriptor. Similarly to the Light Field Descriptor, the proposed method is based on rendering several two-dimensional projections of a 3D model, taken from various points of view. However, the proposed descriptor uses the $2 D$ Polar-Fourier transform for obtained projections This enables the new descriptor to be more efficient in the recognition or retrieval of 3D models. In order to evaluate the performance of the algorithm, it was experimentally compared with four other popular approaches - the Extended Gaussian Image, Shape Distributions, Shape Histogram and Light Field Descriptor - in the problem of 3D shape retrieval. The achieved results have shown that the new method outperforms the other four explored ones. The presented $3 \mathrm{D}$ shape descriptor can be used in representation, recognition and retrieval of $3 \mathrm{D}$ models.
\end{abstract}

Keywords: 3D model retrieval, 3D shape description, Polar-Fourier transform.

\section{Introduction}

The problem of 3D model representation, recognition and retrieval is more popular nowadays than for example ten or twenty years ago. It is mainly caused by the recent development in computer hardware and software and possibility of efficient and fast computation of large multimedia data. Thanks to this many new applications of 3D models have appeared. One of them, especially arising lately, is the $3 \mathrm{D}$ model retrieval, which is a special case of Content-Based Information Retrieval problem of retrieving data from usually large multimedia collections. Nevertheless, from the historical point of view the first popular application of 3D models was the Computer Aided Design, CAD ([1]). However, some other exemplary applications can be easily found. For example, the entertainment application became more common lately ([2]) — in virtual reality, games, movies. Another example is the three-dimensional biometrics, mainly the 3D face recognition ([3]). Finally, the incorporation of a 3D shape description method in the 
MPEG-7 standard for multimedia content description has to be mentioned (4]). In spite of the increasing popularity of $3 \mathrm{D}$ model processing the number of algorithms developed for the representation of 3D shapes is still definitely smaller than for the planar (two-dimensional) ones. However, there have been several approaches developed and explored so far. They can be divided into four main groups: geometrical, structural, symmetrical and local. The approaches based on geometrical information about a model are the oldest and most widely used ones and hence may be easily enumerated. They include the Extended Gaussian Image, EGI ([5]), its modified version - Complex Extended Gaussian Image, CEGI ([6]), 3D moments ([7]), Shape Histograms ([8]), Shape Distributions ([9]) and spherical harmonics ([10]). The Multiresolutional Reeb Graph ([11]) is an exemplary algorithm from the second group. The Reflective Symmetry Descriptor (12 ) belongs to the third one. Finally, the method based on canonical geometric scale-space analysis ([13]) is an example of the local approaches.

In the paper new algorithm is proposed. The general idea applied here is close to the approach used in the Light Field Descriptor (14). Namely, twenty two-dimensional projections for a 3D model are obtained, with cameras placed in the vertices of dodecahedron enclosing the object. However this is the only similarity, because for the achieved projected planar shape another approach for its description is used. This time the $2 D$ Polar-Fourier transform is applied, which is very efficient in the problem of planar shape recognition.

The proposed Polar-Fourier 3D Shape Descriptor was experimentally compared with results provided by four other 3D shape descriptors - the Extended Gaussian Image ([5]), Shape Distributions ([9]), Shape Histograms ([8]) and Light Field Descriptor ([14]). These algorithms selected for comparison were previously analysed in [15]. For the problem of 3D object retrieval the models taken from The Princeton Shape Benchmark database ([16]) were applied.

The remaining part of the paper is organized as follows. Section 2 presents the proposed Polar-Fourier 3D Shape Descriptor. Section 3 describes briefly approaches selected for the experimental comparison with the proposed algorithm. Section 4 provides a detailed description of the conditions and results of the performed experiments. Finally, the last section concludes the paper and provides some suggestions for further research directions.

\section{Description of the Polar-Fourier 3D Shape Descriptor}

As it has been stated in the introductory part of this paper, the motivation underlying the construction of a new 3D shape descriptor was the good result of the Light Filed Descriptor in the 3D model retrieval. The idea is based on the use of another planar shape descriptor for the projected 2D shapes. Hence, the beginning of the proposed algorithm is similar to the previous one.

The Polar-Fourier 3D Shape Descriptor starts with the calculation of the centroid $L$ of a three-dimensional object:

$$
L=\left(L_{x}, L_{y}, L_{z}\right)=\left(\frac{1}{n} \sum_{i=1}^{n} x_{i}, \frac{1}{n} \sum_{i=1}^{n} y_{i}, \frac{1}{n} \sum_{i=1}^{n} z_{i}\right)
$$


where:

$\left(x_{i}, y_{i}, z_{i}\right)$ - a vertex of an object, $n$ - number of vertices for particular 3D shape.

Later, all vertices are translated in order to move the centroid into the origin of the co-ordinate system. For a vertex $P$ it can be formulated as follows:

$$
P_{i}=\left(x_{i}, y_{i}, z_{i}\right)=\left(x_{i}-L_{x}, y_{i}-L_{y}, z_{i}-L_{z}\right),
$$

where: $i=1,2, \ldots, n$.

The normalisation of co-ordinates according to the maximal distance from the centre of gravity is then performed. Thanks to this we can achieve the unitary maximal distance:

$$
M=\max _{i}\left\{\left\|P_{i}-L\right\|\right\}
$$

where: $i=1,2, \ldots, n$.

And:

$$
P_{i}=\left(\frac{x_{i}}{M}, \frac{y_{i}}{M}, \frac{z_{i}}{M}\right)
$$

Later, the projections are obtained from 20 various angles. It results from the assumption that cameras are placed in the vertices of the dodecahedron enclosing the object. Each time the camera is directed into the origin of the co-ordinate system. The projections are stored in bitmaps. For each shape from them polar co-ordinates are calculated for a contour. Firstly, the centroid for the planar shape is derived (notice that the centroid $L$ of the $3 \mathrm{D}$ shape is not equivalent to the centoid $O$, which is calculated for each planar shape separately):

$$
O=\left(O_{p}, O_{q}\right)=\left(\frac{1}{s} \sum_{i=1}^{s} p_{i}, \frac{1}{s} \sum_{i=1}^{s} q_{i}\right) .
$$

where:

$s$ - number of points in a contour of a planar shape,

$p_{i}, q_{i}$ - Cartesian coordinates of the $i$-th point of the projected shape.

By means of the centre of an object $O$ we can calculate the polar co-ordinates - $\Theta^{i}$ for angles and $P^{i}$ for radii:

$$
\begin{gathered}
\rho_{i}=\sqrt{\left(p_{i}-O_{p}\right)^{2}+\left(q_{i}-O_{q}\right)^{2}}, \\
\theta_{i}=\operatorname{atan}\left(\frac{q_{i}-O_{q}}{p_{i}-O_{p}}\right) .
\end{gathered}
$$

The achieved points are put into the matrix, providing as a result a twodimensional representation. This enables us to apply the $2 D$ Fourier transform to it. Usually for the shape representation, the absolute spectrum is used. Its values are derived by means of the following equation ([17]): 


$$
C(k, l)=\frac{1}{H W}\left|\sum_{h=1}^{H} \sum_{w=1}^{W} R(h, w) \cdot e^{\left(-i \frac{2 \pi}{H}(k-1)(h-1)\right)} \cdot e^{\left(-i \frac{2 \pi}{W}(l-1)(w-1)\right)}\right|,
$$

where:

$H, W$ - height and width of the image in pixels,

$k$ - sampling rate in vertical direction $(k \geq 1$ and $k \leq H)$,

$l$ - sampling rate in horizontal direction $(l \geq 1$ and $l \leq W)$,

$C(k, l)$ - value of the coefficient of discrete Fourier transform in the coefficient matrix in $k$ row and $l$ column,

$R(h, w)$ - value in the image plane with coordinates $h, w$.

From the obtained absolute spectrum square subpart with a side equal to 10 elements is selected and concatenated into the vector. It represents a planar projection of a 3D model. For matching any similarity or dissimilarity measure may be applied, e.g. the Euclidean distance.

\section{Brief Description of the Algorithms Selected for the Comparison with the Proposed Method}

As it has already been mentioned in the first section, the proposed algorithm for the 3D shape representation was compared with the results of four other algorithms - Extended Gaussian Image ([5]), Shape Distributions ([9]), Shape Histograms ([8]) and Light Field Descriptor ([14]), provided in [15]. In this section each of them is shortly described.

The Extended Gaussian Image (EGI, [5]) is one of the oldest and most popular techniques for the description of 3D models. In this approach the Gaussian image is obtained through the association of the point on the Gaussian sphere with each point on object's surface with the same surface orientation.

Each point belonging to a patch on the object (denoted as $\delta J$ ) corresponds to a point on the Gaussian sphere (denoted as $\delta S$ ). The Gaussian curvature can be defined as being equal to the limit of the ratio of the two areas as they tend to zero $([5])$ :

$$
K=\lim _{\delta J \rightarrow 0} \frac{\delta S}{\delta J}=\frac{\mathrm{d} S}{\mathrm{~d} J} .
$$

Assuming $S$ - the area of the corresponding patch on the Gaussian sphere, having in mind the previous equation, one can derive the formula ([5]):

$$
\iint_{J} K \mathrm{~d} J=\iint_{S} \mathrm{~d} S=S .
$$

For $J$ denoting the corresponding patch on the object the above formula can be rewritten as $([5])$ :

$$
\iint_{S} \frac{1}{K} \mathrm{~d} S=\iint_{J} \mathrm{~d} J=J
$$


The inverse of the Gaussian curvature in the formulation of the EGI descriptor can be applied thanks to the above relationship ([5]). That gives the possibility of a mapping that associates the inverse of the Gaussian curvature for a point on the surface with a corresponding point on the Gaussian sphere. The EGI can be defined in the following way $([5])$ :

$$
G(\zeta, \eta)=\frac{1}{K(\mu, \nu)},
$$

where point with the coordinates $(\zeta, \eta)$ lies on the Gaussian sphere and has the same normal as point with the coordinates $(\mu, \nu)$ on the original surface.

The Shape Distribution (9]) was the second approach compared with the proposed algorithm. In this method a function representing a model is firstly selected. It may be of any type, however the authors have proposed a few ones: the angle between three random points on the object's surface (A3), the distance between a centroid and a random point on the surface (D1), the distance between a pair of random points (D2), the square root of the area of the triangle lying between three random points on the surface (D3), and the cube root of the volume of the tetrahedron between four random points on the surface (D4).

For a function $N$ samples are evaluated and using them a histogram is constructed, containing the information on how many of the samples fall into $B$ bins. From the histogram a piecewise linear function is derived, with $V$ equally spaced vertices, $V \leq B$, e.g. $N=1024^{2}$ samples, $B=1024$, and $V=64$ vertices (9]).

All polygons of the $3 \mathrm{D}$ object are split into triangles in order to obtain the samples. For each triangle its area is calculated and stored along with the cumulative area of all previously considered triangles. Later, a triangle with a probability proportional to its area is selected. This task is performed through the generation of a random number between 0 and the total cumulative area and performing a binary search on the array of cumulative areas. For each of thus obtained triangles a point $P$ on its surface is derived, applying two random numbers $r_{1}$ and $r_{2}$ ranged from 0 to 1 ([9]):

$$
P=\left(1-\sqrt{r_{1}}\right) A+\sqrt{r_{1}}\left(1-r_{2}\right) B+\sqrt{r_{1}} r_{2} C,
$$

where $A, B$ and $C$ - the vertices of the selected triangle.

The third approach was the Shape Histograms ([8]). The general idea of the algorithm is based on the process of partitioning of the space, where a $3 \mathrm{D}$ object is placed. Using the particular obtained cells the histogram is built. The method of the decomposition of the space can be chosen freely. However, the authors of the approach have proposed three ones - a shell model, a sector model, and a spider-web model (see Fig. 1 for illustration).

The Light Field Descriptor (LFD, 14]) was the last method, which has been compared with the proposed algorithm. In fact its main idea was the basis for the algorithm proposed in this paper - the rendering of several two-dimensional projections of a 3D object (see Fig. 2). Those projections are compared in order to indicate the similar objects. Obviously, this task is performed for various points of view. 


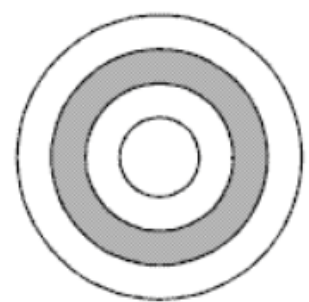

4 shell bins

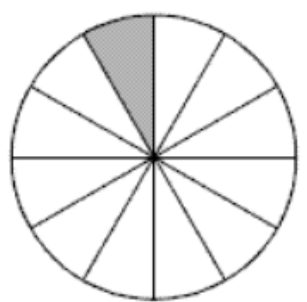

12 sector bins

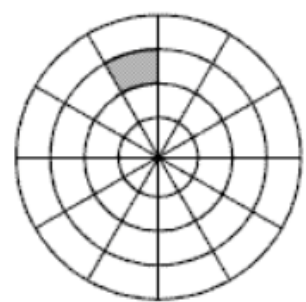

48 combined bins

Fig. 1. Three exemplary methods of space decomposition for shape histograms ([8])
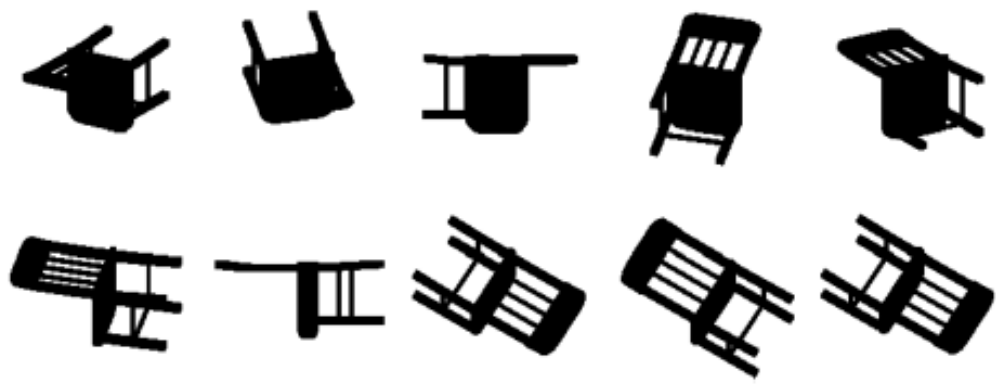

Fig. 2. Exemplary projections obtained for the representation of a model by means of the Light Field Descriptor ([14])

The algorithm for obtaining the $L F D 3 \mathrm{D}$ shape description starts with the shifting of the object's vertices into the origin of the Cartesian co-ordinates system. The second step is the normalization of the co-ordinates according to the maximal one. Later, the crucial stage starts - for 20 various angles (the cameras are placed in the vertices of dodecahedron enclosing the model) the rendered planar projections are obtained ([14]). The obtained planar projections of 3D object are stored in bitmaps, and they are the representations of the model. Hence, the similarity between two objects is calculated by means of matching between their projections.

\section{Experimental Conditions and Results}

All five 3D shape description algorithms presented in this paper - the proposed Polar-Fourier 3D Shape Descriptor as well as the four approaches selected for the comparison - were experimentally evaluated by means of the Princeton Shape Benchmark ([16]). It is a free database made by Princeton University, created to help in performing the benchmark of different algorithms. This database is very popular in evaluating the 3D shape descriptors (see for example [18] and [19]).

During the experiments 312 objects belonging to 13 different classes were used (see Fig. 3). The idea of the experiment was simple. The retrieval was 

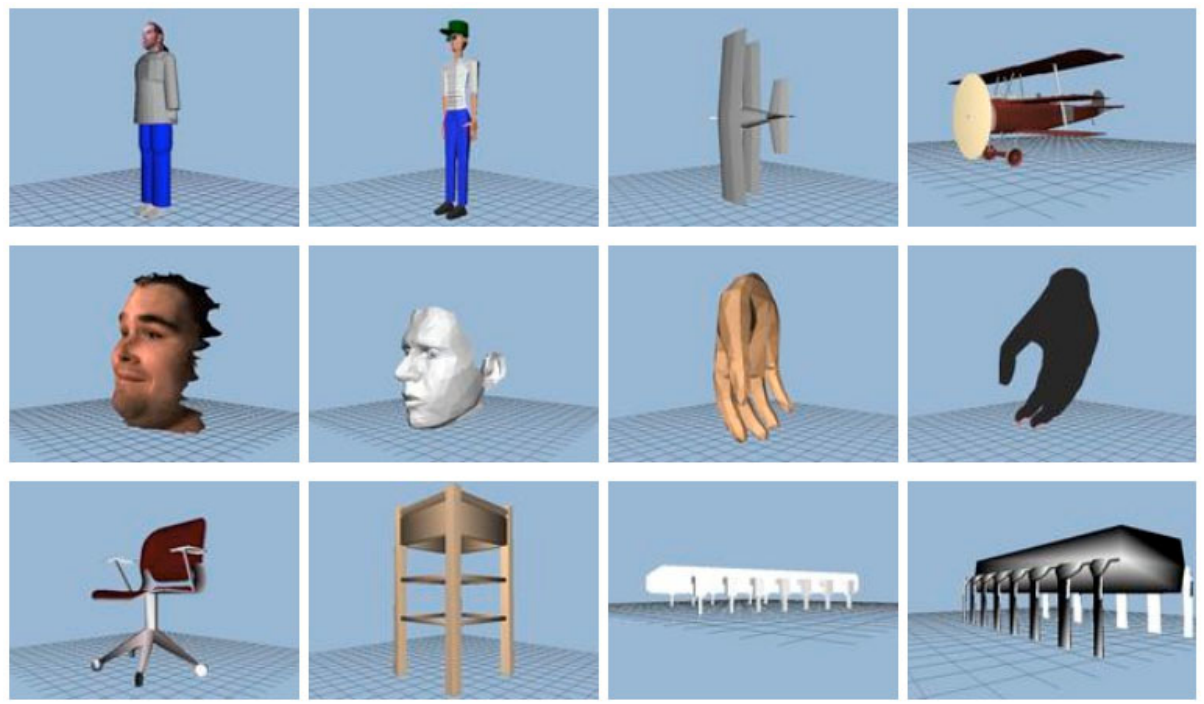

Fig. 3. Examples of the 3D models used in the experiment, taken from the Princeton Shape Benchmark database ([16])

successful if the Euclidean distance between a represented using a descriptor test and template was the smallest for objects belonging to the same class. Obviously, the template models did not perform the role of the test ones. The precise results of the retrieval obtained for investigated algorithms are provided in Table 1 .

The results provided in Table@prove that the proposed algorithm outperforms the other explored 3D shape description techniques. Its average recognition rate (RR) is close to $75 \%$. This result is more than $5 \%$ better than in the case of the second best descriptor - Light Field Descriptor.

Although the average result of the Polar-Fourier $3 D$ Shape Descriptor is significantly the highest in some cases other methods have performed better. For example, $E G I$ achieved $80 \%$ for class number 5 , while the $P-F 3 D$ was two times worse. Similarly, for class no. $12 E G I$ achieved $50 \%$, while the proposed method gave $37.5 \%$. The highest difference is visible in the case of class no. 13. EGI descriptor worked in that case really well, while $P-F 3 D$ achieved only a $33.33 \%$ retrieval rate. On the other hand, for the rest of the classes the proposed approach has performed better.

Shape Distributions proved better than the Polar-Fourier $3 D$ only in one case. For class no. 3 the SD achieved $84.21 \%$ and the $P-F 3 D-63.16 \%$. The Light Field Descriptor has also appeared better only once. Its RR for class no. 6 was equal to $88.89 \%$, while the $P-F 3 D$ was slightly worse and achieved $83.33 \%$. Shape Histograms turned out the worst during the test and never gave a result better than the proposed approach. 
Table 1. Results of the experiments - percentage of the successful retrieval (retrieval rate) for particular $3 \mathrm{D}$ shape descriptors

\begin{tabular}{lccccc}
\hline Class no. & EGI & SH & SD & LFD & P-F 3D \\
\hline 1. & 57.75 & 29.58 & 78.87 & 78.87 & 83.10 \\
2. & 65.71 & 48.57 & 57.14 & 85.71 & 85.71 \\
3. & 52.63 & 21.05 & 84.21 & 57.89 & 63.16 \\
4. & 53.13 & 56.25 & 34.38 & 56.25 & 62.50 \\
5. & 80.00 & 20.00 & 30.00 & 10.00 & 40.00 \\
6. & 50.00 & 44.44 & 72.22 & 88.89 & 83.33 \\
7. & 66.67 & 33.33 & 50.00 & 50.00 & 66.67 \\
8. & 66.67 & 0.00 & 0.00 & 33.33 & 66.67 \\
9. & 65.12 & 67.44 & 27.91 & 74.42 & 76.74 \\
10. & 70.00 & 10.00 & 60.00 & 60.00 & 70.00 \\
11. & 60.61 & 9.09 & 54.55 & 66.67 & 75.76 \\
12. & 50.00 & 12.50 & 12.50 & 25.00 & 37.50 \\
13. & 100.00 & 16.67 & 50.00 & 16.67 & 33.33 \\
\hline Overall & $\mathbf{6 0 . 2 6}$ & $\mathbf{3 6 . 8 6}$ & $\mathbf{5 6 . 0 9}$ & $\mathbf{6 8 . 9 1}$ & $\mathbf{7 4 . 6 8}$ \\
\hline
\end{tabular}

The result of the Polar-Fourier 3D Shape Descriptor seems to be far from the ideal; however, the problem of $3 \mathrm{D}$ model retrieval is very difficult. This is illustrated in Fig. 4, where some examples of the objects from the same class are presented - they sometimes look very dissimilar.
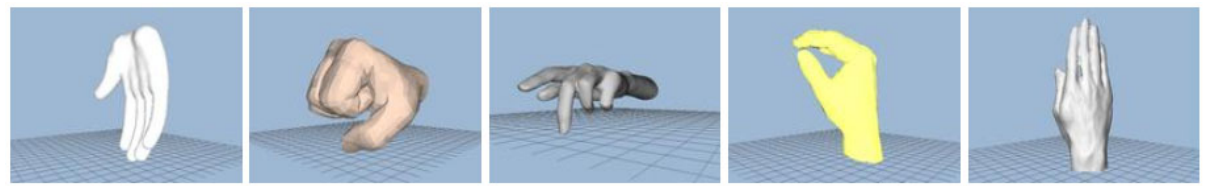

Fig. 4. Illustration of the difficulty involved with 3D-model retrieval - examples of $3 \mathrm{D}$ shapes belonging to the same class, yet very different in appearance ([16])

\section{Conclusions and Future Plans}

In the paper a new algorithm for the description of three-dimensional shapes has been presented and experimentally compared with four popular methods - Extended Gaussian Image (EGI, 5]), Shape Distributions (9]), Shape Histograms (8]) and Light Field Descriptor (LFD, 14]). The problem of 3D object retrieval has been analysed. For this purpose the models from the Princeton Shape Benchmark ([16]) were used. The achieved average retrieval rates have indicated that the proposed method works better in the problem than the other approaches. The Polar-Fourier 3D Shape Descriptor achieved almost a $75 \%$ retrieval rate. This result can be considered as satisfactory, because in many cases the objects within a class display strong differences (see Fig. 4 for an example). 
The other algorithms achieved a retrieval rate equal to: 69\% (Light Field Descriptor), 60\% (Extended Gaussian Image), 56\% (Shape Distributions), and 37\% (Shape Histograms).

The obtained experimental results have confirmed the high efficiency of the approach proposed in Light Field Descriptor ([14]), namely rendering 2D projections of a 3D model, taken from various points of view. The improvement applied in the new descriptor is based on the usage of polar-Fourier transform for the achieved planar shapes. In the future, some other $2 \mathrm{D}$ shape descriptors will be verified by means of the same method. It is possible that thanks to them the retrieval results will be even better.

\section{References}

1. Ikeuchi, K.: Generating an interpretation tree from a CAD model for 3D-object recognition in bin-picking tasks. International Journal of Computer Vision 1(2), 145-165 (1987)

2. Lengyel, E.: Mathematics for 3D Game Programming and Computer Graphics, 2nd edn. Charles River Media (2003)

3. Bronstein, A.M., Bronstein, M.M., Kimmel, R.: Expression-Invariant 3D Face Recognition. LNCS, vol. 2688, pp. 62-70 (2003)

4. Bober, M.: MPEG-7 Visual Shape Descriptors. IEEE Trans. on Circuits and Systems for Video Technology 11(6), 716-719 (2001)

5. Horn, B.: Extended Gaussian Images. Proc. of the IEEE A.I. Memo, no. 740 72(12), 1671-1686 (1984)

6. Kang, S., Ikeuchi, K.: Determining 3-D Object Pose Using the Complex Extended Guassian Image. In: Proc. of the CVPR, pp. 580-585 (1991)

7. Novotni, M., Klein, R.: Shape Retrieval Using 3D Zernike Descriptors. ComputerAided Design 36(11), 1047-1062 (2004)

8. Ankerst, M., Kastenmuller, G., Kriegel, H., Seidl, T.: 3D Shape Histograms for Similarity Search and Classification in Spatial Databases. In: Proc. of the 6th Int. Symp. on Spatial Databases, pp. 207-226 (1999)

9. Osada, R., Funkhouser, T., Chazelle, B., Dobkin, D.: Matching 3D Models with Shape Distributions. In: Proc. of Int. Conf. SMI 2008, pp. 154-166 (2001)

10. Mousa, M.-H., Chaine, R., Akkouche, S., Galin, E.: Toward an Efficient TriangleBased Spherical Harmonics Representation of 3D Objects. Computer Aided Geometric Design 25(8), 561-575 (2008)

11. Hilaga, M., Shinagawa, Y., Kohmura, T., Kunii, T.L.: Topology Matching for Fully Automatic Similarity Estimation of 3D Shapes. In: Proc. of the 28th Conference on Computer Graphics and Interactive Techniques, pp. 203-212 (2001)

12. Kazhdan, M., Chazelle, B., Dobkin, D., Funkhouser, T., Rusinkiewicz, S.: A Reflective Symmetry Descriptor for 3D Models. Algorithmica 38, 201-225 (2003)

13. Novatnack, J., Nishino, K.: Scale-Dependent/Invariant Local 3D Shape Descriptors for Fully Automatic Registration of Multiple Sets of Range Images. In: Forsyth, D., Torr, P., Zisserman, A. (eds.) ECCV 2008, Part III. LNCS, vol. 5304, pp. 440-453. Springer, Heidelberg (2008)

14. Chen, D.-Y., Ouhyoung, M., Tian, X.-P., Shen, Y.-T.: On visual similarity based 3D model retrieval. Computer Graphics Forum, 223-232 (2003) 
15. Frejlichowski, D.: 3D Shape Description Algorithms Applied to the Problem of Model Retrieval. Central European Journal of Engineering 1(1), 117-121 (2011)

16. Shilane, P., Min, P., Kazhdan, M. M., Funkhouser, T.A.: The Princeton Shape Benchmark. In: Proc. of the SMI 2004, Genova, Italy, pp. 145-156 (2004)

17. Kukharev, G., Kuzminski, A.: Biometric Techniques Part I - Face Recognition Methods. Szczecin University of Technology Press, Szczecin (2003) (in polish)

18. Min, P., Kazhdan, M., Funkhouser, T.: A Comparison of Text and Shape Matching for Retrieval of Online 3D Models. In: Heery, R., Lyon, L. (eds.) ECDL 2004. LNCS, vol. 3232, pp. 209-220. Springer, Heidelberg (2004)

19. Grana, C., Davolio, M., Cucchiara, R.: Similarity-Based Retrieval with MPEG-7 3D Descriptors: Performance Evaluation on the Princeton Shape Benchmark. In: Thanos, C., Borri, F., Candela, L. (eds.) Digital Libraries: Research and Development. LNCS, vol. 4877, pp. 308-317. Springer, Heidelberg (2007) 